\title{
Low-Cost MIMO Radio Over Fiber System for Multi-Service DAS Using Double Sideband Frequency Translation
}

\author{
Yumeng Yang, Student Member, IEEE, Michael J. Crisp, Member, IEEE, \\ Richard V. Penty, Senior Member, IEEE and Ian H. White, Fellow, IEEE
}

\begin{abstract}
In this paper, a novel low-cost DSB frequency translation system is experimentally demonstrated and its theory is mathematically proved. The new system is capable of transmitting wideband LTE MIMO signals and supporting multiple services. Experimentally, $2 \times 2$ LTE MIMO channels with $20 \mathrm{MHz}$ bandwidth, and a $700 \mathrm{MHz}$ carrier frequency, are transmitted simultaneously along with an IEEE 802.11g signal (54Mbps) over a $300 \mathrm{~m}$ length of multi-mode fiber (MMF). The MIMO channel matrix of the system is retrieved and the condition number is calculated. It can be seen in the experimental results that the system is well conditioned, has a low error vector magnitude (EVM) and the transmission of the MIMO signals has negligible effect on the IEEE 802.11g signal. The use of a preamplifier minimizes the non-linearity introduced by the frequency mixers, resulting in a high spurious-free dynamic range (SFDR). Compared to a single sideband (SSB) system, where multiple filters are used and two channels encounter different EVMs, the new DSB system avoids unnecessary filtering and the impairments introduced will be the same for both channels. Therefore it can be concluded that the improved MIMO over fiber system using DSB frequency translation technology is a potential low-cost solution for multiservice MIMO-enabled distributed antenna systems (DAS).
\end{abstract}

Index Terms-Indoor radio communication, distributed antenna system (DAS), radio-over-fiber (ROF), optical fiber, multiplexing, multiple-input-multiple-output (MIMO)

\section{INTRODUCTION}

$\mathrm{W}$ IRELESS communication has revolutionized many lives and is still experiencing fast growth. Currently, there are 6.9 billion mobile cellular subscriptions worldwide [1]. As 4G mobile deployment is growing rapidly, a huge amount of mobile data is now being generated and indeed it is predicted that by $2018,4 \mathrm{G}$ will make up $15 \%$ of connections and $51 \%$ of total traffic [2]. In the $4 \mathrm{G}$ standards, such as long term evolution

Manuscript received xx, $\mathrm{xx}, \mathrm{xxxx}$. This work was supported in part of the UK EPSRC via the COPOS II grant and the European Community via the FP7 programme Quaternian project.

Additional data related to this publication is available at the http://www.repository.cam.ac.uk/handle/1810/250308 data repository.

The authors are with the Electrical Engineering Division, Department of Engineering, University of Cambridge, Cambridge, Cambridgeshire CB3 OFA, U.K. (e-mail: yy371@cam.ac.uk; mjc87@cam.ac.uk; rvp11@cam.ac.uk; ihw3@cam.ac.uk).

Copyright (c) 2015 IEEE. Personal use of this material is permitted. However, permission to use this material for any other purposes must be obtained from the IEEE by sending a request to pubs-permissions@ieee.org.
(LTE) and LTE-advanced, the use of MIMO techniques can increase data throughput and improve transmission reliability without increasing occupied spectrum. This is particularly important in indoor environments, where $80-90 \%$ of wireless data traffic originates [3], but propagation conditions are usually poor with a high degree of multipath.

The DAS is one of the most popular methods to increase indoor wireless signal coverage. Many indoor DAS systems use RoF technology to transmit signals between a base station (BS) and remote antenna units (RAUs), for versatility, flexibility, scalability and low cost [4].

Fig.1 shows a typical layout of MIMO-enabled DAS system. In most building infrastructure, an optical fiber for Ethernet communication is installed [5]. Making use of the existing optical fiber to transmit multiple MIMO signal streams to each RAU can thus be a cost-effective way to build a MIMO-enabled DAS system. In this paper, we propose a low cost multi-service MIMO-enabled RoF system, which can transmit multiple wide-band LTE MIMO-type signals simultaneously with a separate SISO radio service (which for ease of implementation is chosen to be Wi-Fi) over a single optical fiber. Similar to the traditional frequency translation system [6][7], the proposed system can be easily extended from $2 \times 2$ layout to higher order MIMO simply by adding additional local oscillators (LOs) [7]. In the system with $2 \mathrm{n}+1$ MIMO channels, $\mathrm{n}$ pairs of local oscillators are required.

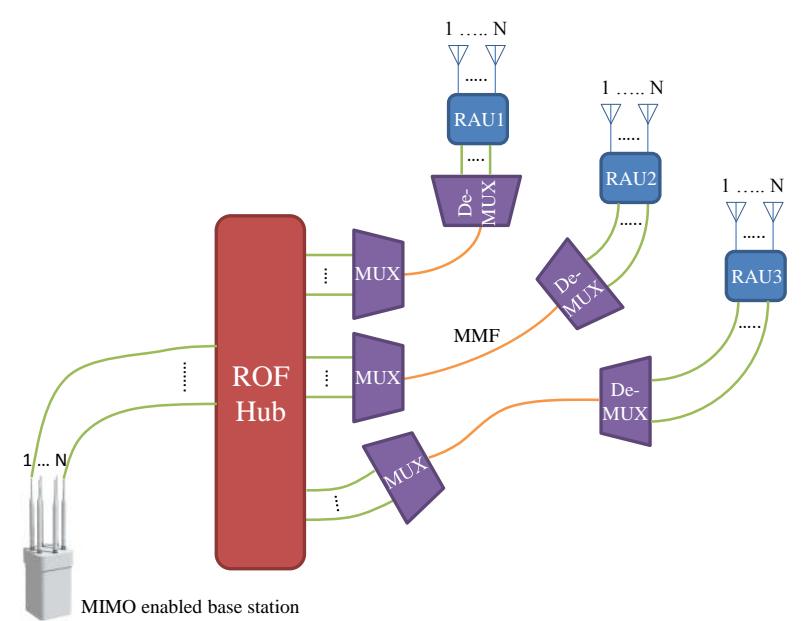

Fig. 1. The layout of $N \times N$ MIMO-enabled ROF DAS, which feeds each RAU with multiple MIMO signals 


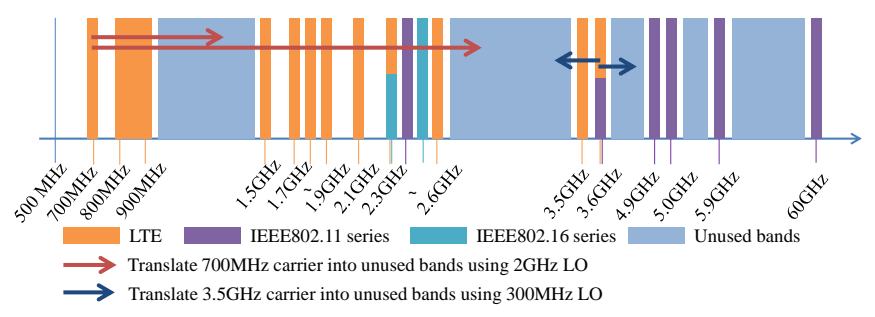

Fig.2. Global LTE and Wi-Fi spectrum allocation and schematic of frequency translation

\section{MIMO-ENABLED ROF}

\section{A. Multiplexing Schemes for MIMO-Enabled ROF}

As MIMO signal channels overlap in the frequency domain, a multiplexing scheme is required to transmit them simultaneously to the remote antenna unit. A straight forward solution to tackle the problem is to install as many optical fibers as the number of MIMO channels. However, this leads to high optical component and fiber installation cost.

Some solutions, including wavelength division multiplexing (WDM) [8], subcarrier multiplexing (SCM) [9] and mode division multiplexing (MDM) [10], are capable of transmitting MIMO signals over a single optical fiber, however, they can be expensive and complicated to implement. In a WDM system, an optical source and photodetector is required for each MIMO channel. In an SCM system, n-1 LOs are required for n MIMO signal channels. In other words, the number of optical or electrical components increases linearly with the number of MIMO signal channels in WDM systems and SCM systems. In an MDM system, an expensive spatial light modulator is required.

Digital time division multiplexing (TDM) can also be a potential solution. However, here radio frequency signals need to be digitized before transmission and this requires high speed digital signal processing modules, which add cost and significant power consumption. Although some research has been done on lowering the digitized ROF bit rate [11], high cost components, such as analog-to-digital and digital-to-analog converters, are unavoidable.

To lower the cost of MIMO-enabled ROF system, two alternative analog ROF systems using double sideband (DSB) frequency translation [6] and single sideband (SSB) frequency translation [7] techniques have been proposed by researchers. The operating principle of these two systems is similar to a traditional SCM system, but here two MIMO channels share the same LO, so that the number of LOs can be halved. However, in [6] and [7], the authors find that the DSB system does not have such good performance as the SSB system and cannot work when the upper band and the lower band of the translated signal are widely separated. Moreover, a narrow band notch filter is required in the DSB frequency translation system which results system to only carrying a single service.

Some researchers have improved the SSB system to support multiple services by translating signals to different bands [12] and lower its cost using directly modulated lasers and MMF [13]. However, due to the filtering process required by the SSB frequency translation system, it unavoidably wastes the power of one of the upper sideband and the lower sideband of the signal and it requires many filters at the basestation side and the RAU side of the system.

There are also researchers working on low-cost MDM systems [10], WDM systems [14,15], as well as polarization-multiplexing systems [16]. Although all these systems can support multiple-service, none of them is likely to fundamentally be lower cost than the system using frequency translation technique.

\section{B. A Novel Low-Cost MIMO-Enabled RoF Technique}

In this paper, an improved MIMO-enabled RoF system using DSB frequency translation is proposed. Here as shown in Fig. 2, a $2 \mathrm{GHz}$ LO translates both LTE MIMO signals to unused frequency bands $(1 \mathrm{GHz}-1.4 \mathrm{GHz}$ and $2.7 \mathrm{GHz}-3.4 \mathrm{GHz})$. The demonstrated system works for $700 \mathrm{MHz}$ LTE signals. For higher LTE carrier frequencies, $3.7 \mathrm{GHz}-4.9 \mathrm{GHz}$ and $5.0 \mathrm{GHz}$ to $5.9 \mathrm{GHz}$ bands will need to be used, requiring a high bandwidth ROF link. The capability of translating the original LTE signal into unused frequency bands allows multi-service operation of the system.

Compared to the system using SSB frequency translation, where the performance of the higher frequency MIMO channel can be limited by the optical link bandwidth, in the DSB frequency translation system, as the two MIMO channels are orthogonally multiplexed, both MIMO channels have the same transmission performance and thus higher effective EVM dynamic range.

Furthermore, in the DSB frequency translation system, the mixers at the RAU side make use of both the upper band and the lower band of the translated signal. Since the signal is added coherently, but the noise in each side band is added incoherently $[6,7]$, the noise figures (NF) of the system can be reduced by the mixers at the RAU side.

Compared to the traditional DSB frequency translation system, which can only transmit narrow band signals and which is therefore not compatible with a commercial LTE service, in the proposed system, a simple power equalizer is used to compensate the frequency response of the optical link, so that the system is capable of transmitting wideband LTE and Wi-Fi signals. A pre-amplifier with a suitable gain is used to minimize the system accumulated 3rd order output intercept point (OIP3) at the BS side with minimal increase of the system noise floor in order to maximize the system dynamic range performance.

\section{THEORY}

Using the DSB frequency translation technique, two MIMO channels are orthogonally multiplexed with each other. At the BS side, the LO signal is split using a $90^{\circ}$ hybrid coupler to drive two mixers. At the RAU side, a de-multiplexer is built. After mixing down and applying a bandpass filter, the original MIMO signals can be recovered if the LO at the RAU side is adjusted to a suitable phase $(\theta)$. Here, as the upper band and the lower band have already been combined, a bandpass filter does not lower the signal to noise ratio (SNR). The multiplexing and de-multiplexing procedure is illustrated in Fig.3, equations (1) to (14) and table I. The RoF link is modeled by a low-pass filter (LPF) and time delay. 


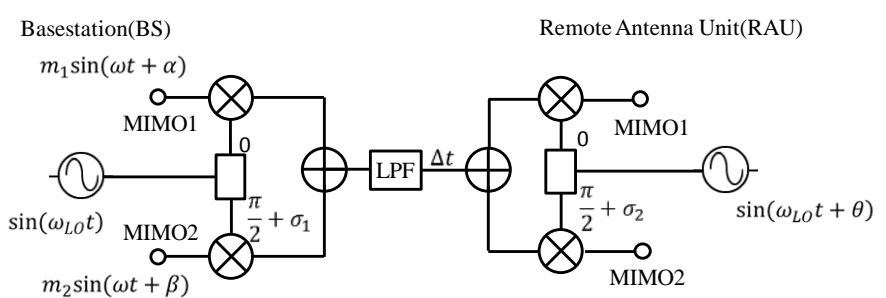

The optical link is acting as a low-pass filter.

Fig. 3. Schematic of the model of MIMO-enabled ROF system using DSB frequency translation.

$$
\begin{aligned}
M_{\text {mux }}= & m_{1} \sin (\omega \mathrm{t}+\alpha) \sin \left(\omega_{L O} \mathrm{t}\right) \\
& \quad+\quad m_{2} \sin (\omega \mathrm{t}+\beta) \cos \left(\omega_{L O} \mathrm{t}+\sigma_{1}\right) \\
= & M_{\text {pre }, \omega-\omega_{L O}}(t)+M_{\text {pre }, \omega+\omega_{L O}}(t)
\end{aligned}
$$

in which,

$$
\begin{aligned}
M_{\text {pre }, \omega-\omega_{L O} O}(\mathrm{t})=\frac{1}{2}\left\{m_{1} \cos \left[\left(\omega-\omega_{L O}\right) t+\alpha\right]\right. \\
\left.\quad+m_{2} \sin \left[\left(\omega-\omega_{L O}\right) t+\beta-\sigma_{1}\right]\right\} \\
M_{\text {pre }, \omega+\omega_{L O} O}(t)=\frac{1}{2}\left\{-m_{1} \cos \left[\left(\omega+\omega_{L O}\right) t+\alpha\right]\right. \\
\left.+m_{2} \sin \left[\left(\omega+\omega_{L O}\right) t+\beta+\sigma_{1}\right]\right\}
\end{aligned}
$$

After the ROF link,

$$
\begin{aligned}
& M_{\text {post }, \omega-\omega_{L O}}(\mathrm{t})=G_{\omega-\omega_{L O} O} M_{\text {pre }, \omega-\omega_{L O}}(\mathrm{t}+\Delta \mathrm{t}) \\
& M_{\text {post }, \omega+\omega_{L O}}(\mathrm{t})=G_{\omega+\omega_{L O}} M_{\text {pre } \omega+\omega_{L O}}(\mathrm{t}+\Delta \mathrm{t})
\end{aligned}
$$

Then, the de-multiplexed signal,

$$
\begin{array}{r}
M_{\text {demux } 1}=\left[M_{\text {post }, \omega-\omega_{L O}}(\mathrm{t})+M_{\text {post }, \omega+\omega_{L O}}(\mathrm{t})\right] \\
\cdot \sin \left(\omega_{L O} \mathrm{t}+\theta\right) \\
M_{\text {demux } 2}=\left[M_{\text {post }, \omega-\omega_{L O}}(\mathrm{t})+M_{\text {post }, \omega+\omega_{L O}}(\mathrm{t})\right] \\
\cdot \cos \left(\omega_{L O} \mathrm{t}+\theta+\sigma_{2}\right)
\end{array}
$$

Consider only the components at $\omega$ in channel 1 ,

$$
\begin{aligned}
M_{\text {demux } 1}= & M_{\text {signal } 1}+M_{\text {crosstalk } 1} \\
M_{\text {signal } 1}= & \frac{m_{1} G_{\omega-\omega_{L O}}}{4} \sin \left(\omega t+\alpha+\delta_{1}+\theta\right) \\
& +\frac{m_{1} G_{\omega+\omega_{L O}}}{4} \sin \left(\omega t+\alpha+\delta_{2}-\theta\right) \\
M_{\text {crosstalk } 1}= & -\frac{m_{2} G_{\omega-\omega_{L O}}}{4} \cos \left(\omega t+\beta-\sigma_{1}+\delta_{1}+\theta\right) \\
& +\frac{m_{2} G_{\omega+\omega_{L O}}}{4} \cos \left(\omega t+\beta+\sigma_{1}+\delta_{2}-\theta\right)
\end{aligned}
$$

\begin{tabular}{|c|c|}
\hline Symbol & Quantity \\
\hline $\mathrm{m}_{1}, \mathrm{~m}_{2}$ & Message contained in MIMO channel 1 and channel 2 \\
\hline$\omega$ & Angle frequency of MIMO signals \\
\hline$\omega_{\mathrm{LO}}$ & Angle frequency of LO signal \\
\hline$\alpha, \beta$ & Initial phase of MIMO channel 1 and channel 2 signal \\
\hline$\sigma_{1}, \sigma_{2}$ & $\begin{array}{l}\text { Phase error of the } 90 \text { degree splitter at the BS and the } \\
\text { RAU side }\end{array}$ \\
\hline$\theta$ & Phase difference between two LOs \\
\hline$\Delta \mathrm{t}$ & Delay time between the BS side and RAU side \\
\hline$\delta_{1}, \delta_{2}$ & Respectively $\left(\omega-\omega_{\mathrm{LO}}\right) \Delta \mathrm{t}$ and $\left(\omega+\omega_{\mathrm{LO}}\right) \Delta \mathrm{t}$ \\
\hline $\mathrm{M}_{\text {mux }}$ & $\begin{array}{l}\text { Multiplexed signal, before the laser, but after the } \\
\text { power combiner at the BS side. }\end{array}$ \\
\hline $\mathrm{M}_{\mathrm{pre}, \omega \pm \omega_{\mathrm{LO}}}$ & Frequency component at $\omega \pm \omega_{\mathrm{LO}}$ of $\mathrm{M}_{\operatorname{mux}}$ \\
\hline$M_{\text {post }, \omega \pm \omega_{\text {LO }}}$ & $\begin{array}{l}\text { Frequency component at } \omega \pm \omega_{\text {LO }} \text { of the signal after } \\
\text { optical link (low-pass filter) }\end{array}$ \\
\hline $\mathrm{G}_{\omega \pm \omega_{\mathrm{LO}}}$ & Gain of the optical link (low-pass filter) at $\omega \pm \omega_{\mathrm{LO}}$ \\
\hline$M_{\text {demux1 }}$ & $\begin{array}{l}\text { De-multiplexed signal of channel } 1 \text { and channel } 2 \text { after } \\
\text { the frequency mixer at RAU side }\end{array}$ \\
\hline $\mathrm{M}_{\text {signal1 }}$ & Effective signal contained in $\mathrm{M}_{\text {demux1 }}$ \\
\hline $\mathrm{M}_{\text {crosstalk1 }}$ & Crosstalk contained in $\mathrm{M}_{\mathrm{demux} 1}$ \\
\hline
\end{tabular}

If no crosstalk, $M_{\text {crosstalk } 1}=0$. Then,

$$
\begin{gathered}
\beta-\sigma_{1}+\delta_{1}+\theta=\beta+\sigma_{1}+\delta_{2}-\theta+2 n \pi \\
\text { or } \theta=\sigma_{1}+\omega_{\mathrm{LO}} \Delta \mathrm{t}+n \pi \\
G_{\omega-\omega_{L O}}=G_{\omega+\omega_{L O}}
\end{gathered}
$$

Similarly, if consider channel 2 , when no crosstalk,

$$
\begin{array}{r}
\theta=\omega_{\mathrm{LO}} \Delta \mathrm{t}-\sigma_{2}+n \pi \\
\text { and } G_{\omega-\omega_{L O}}=G_{\omega+\omega_{L O}}
\end{array}
$$

Equations (11) - (14) show that there are three major factors that contribute to the crosstalk: (i) phase mismatch caused by the microwave transmission time $(\Delta t)$; (ii) phase error of the 90-degree hybrid couplers $\left(\sigma_{1}, \sigma_{2}\right)$; (iii) amplitude imbalance between two bands $\left(\mathrm{G}_{\omega-\omega_{\mathrm{LO}}}, \mathrm{G}_{\omega+\omega_{\mathrm{LO}}}\right)$. In order to eliminate the
TABLE I

SYMBOLS USED IN EQUATIONS

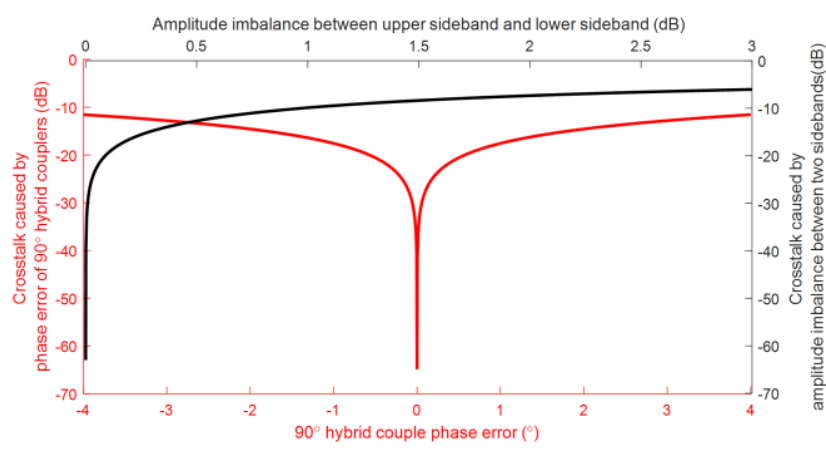

(a)

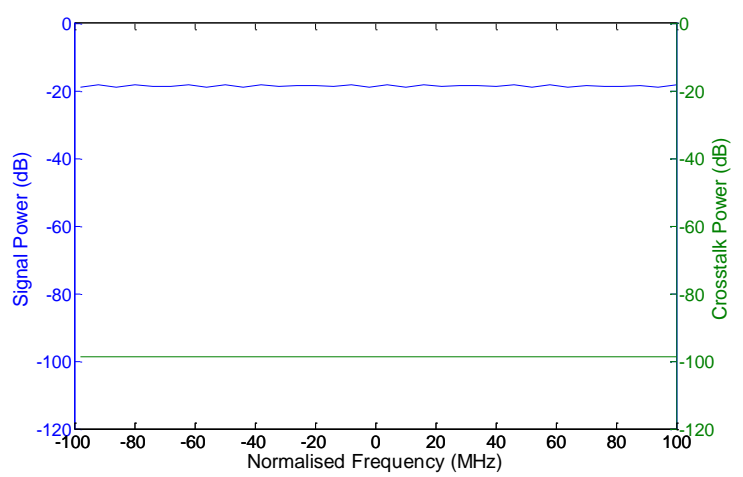

(b)

Fig. 4. (a) Simulation of the crosstalk caused by the phase error of 90 degree hybrid couplers, and amplitude imbalance between two side bands. (b) Simulation result of compensated signal power and crosstalk power at the RAU side vs normalized carrier frequency.

crosstalk between two channels, equations (11)-(14) have to be satisfied.

Due to the group delay of the link $(\Delta t)$, the upper band and the lower band arrive at the frequency mixers with difference phases $\left(\delta_{1}\right.$ and $\left.\delta_{2}\right)$. If there is no phase error in the 90 degree hybrid couplers $\left(\sigma_{1}=0, \sigma_{2}=0\right)$, the effect of $\delta_{1}$ and $\delta_{2}$ can be completely eliminated by adjusting the phase difference between the mux and demux LOs via a delay line, which sets the value of $\theta=\omega_{\mathrm{LO}} \Delta \mathrm{t}+n \pi$. 
However, limited by the phase error of the 90-degree hybrid couplers, equation (11) and (13) can never be fully satisfied at the same time and this becomes one of the major factors which result in the system crosstalk.

The amplitude imbalance between the upper band and the lower band is another important factor that causes crosstalk. As shown in equation (10), when the signals are demultiplexed at the RAU side, the signal from the unwanted channel has to be cancelled which requires that the two sidebands of the DSB signal have the same amplitude.

The simulation results in Fig. 4(a) shows the crosstalk caused by the phase error of the 90-degree hybrid coupler and the amplitude imbalance between two frequency bands. Typically, the phase error of a 90-degree hybrid coupler can be within $2^{\circ}$, but amplitude imbalance of the two sidebands caused by the vibration in the S21 frequency response of the optical link can be $>1 \mathrm{~dB}$. Thus, the amplitude imbalance between two sidebands is the dominant factor contributing to system crosstalk. Fig. 4(b) shows the simulation results of the link with $200 \mathrm{MHz}$ bandwidth MIMO signal. It can be seen that when the value of $\theta$ is chosen to a suitable value and the link has perfectly flat amplitude-frequency response, at the RAU side, good signal power and low crosstalk can be achieved.

In a real RoF link, the amplitude-frequency response can never be perfectly flat and will depend on the response of the laser diode, the multimode fiber, the photodiode, the power amplifier gain, and the frequency mixers at the BS side. In order to return the upper and lower bands to the same amplitude, a simple power equalizer needs to be implemented.

\section{EXPERIMENTAL SET-UP}

Fig.5 shows the experimental layout of the $2 \times 2$ MIMO-enabled ROF system using the DSB frequency translation technique. At the BS side, two MIMO signal channels are mixed using the same local oscillator, but with a $90^{\circ}$ phase difference between them. The resulting quadrature signals are combined with an IEEE $802.11 \mathrm{~g}$ signal using a $3 \mathrm{~dB}$ power combiner. Due to the availability of the equipment, signals are then directly modulated to a DFB laser (3dB RF bandwidth $=2 \mathrm{GHz}$ ), emitting at $\sim 1550 \mathrm{~nm}$ wavelength, although a $1310 \mathrm{~nm}$ wavelength DFB laser may be more practical. In order to achieve an optimum EVM dynamic range, the IEEE $802.11 \mathrm{~g}$ signal is attenuated at the input of the system so that the IEEE 802.11g signal has the same power as each channel of the MIMO signals before direct modulation of laser diode such that the three channels experience the same level of distortion.

A 300m length of OM1 62.5/125nm multi-mode fiber (MMF) has been used to separate the transmitter from the RAU and to be representative of a worst case inbuilding fiber run. If a single-mode fiber (SMF) is used, the system would have better EVM performance, because the dispersion in SMF is much lower than in MMF. At the RAU side, the signal is detected by a PIN photodiode with an integrated transimpedance amplifier (TIA). A simple power equalizer is used to equalize the amplitude of the upper band and the lower band of the mixed

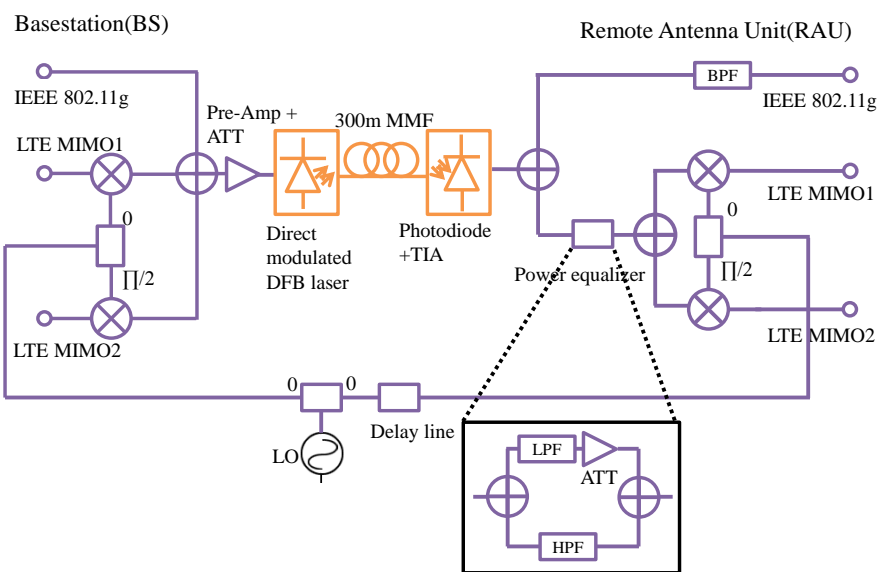

Fig.5. Experimental layout of MIMO-enabled ROF system using DSB frequency translation technique.

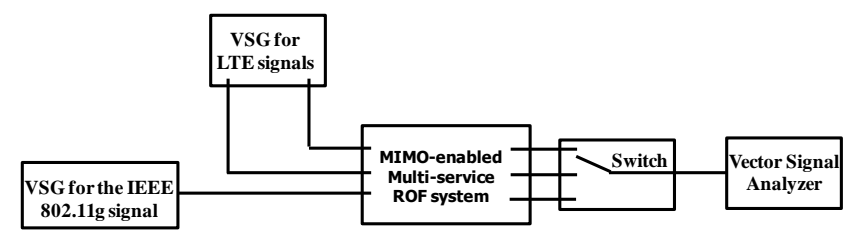

Fig.6. Experimental setup for EVM test.

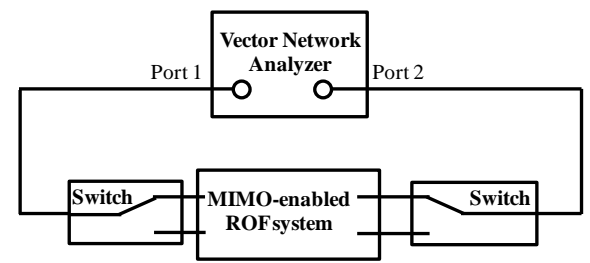

Fig.7. Experimental setup for condition number test.

signals to improve the signal quality after de-multiplexing. Here, the filters in the power equalizer also prevent the leakage of IEEE $802.11 \mathrm{~g}$ signal to the frequency mixers. Following the power equalizer, a similar layout to that at the transmitting side is used to de-multiplex the MIMO signals.

In this experiment, the BS side and the RAU side share the same LO for experimental simplicity. In reality, there should be two LOs, one for each of the BS and the RAU along with a means to ensure that they are locked to the same frequency, such as a phase lock loop (PLL) [17]. To provide the necessary phase shift at the demultiplexer, an RF delay line is used.

Fig.6 shows the experimental layout for the EVM test. A Rohde\&Schwarz ${ }^{\circledR}$ SMW200A vector signal generator and an Anritsu ${ }^{\circledR}$ MS2690A signal generator are used to generate two LTE channels centered at $700 \mathrm{MHz}$ to emulate the independent MIMO channels and an IEEE 802.11g channel (centered at $2.4 \mathrm{GHz}$ ). A Rohde\&Schwarz ${ }^{\circledR}$ FSQ26 vector signal analyzer is used to analyze the de-multiplexed MIMO signals and received $\mathrm{Wi}-\mathrm{Fi}$ signal at the RAU side separately. Limited by the available test equipment, no MIMO processing is performed. It is expected that such processing could result in a lower the EVM by reversing some crosstalk as is illustrated in Section V. The system is tested with $3 \mathrm{MHz}, 10 \mathrm{MHz}$ and $20 \mathrm{MHz}$ bandwidth LTE signals and test results are shown in Section V.

The experimental setup for the condition number measurement is shown in Fig. 7. A vector network analyzer (VNA) scans from $600 \mathrm{MHz}$ to $1 \mathrm{GHz}$ and 1600 points are taken 


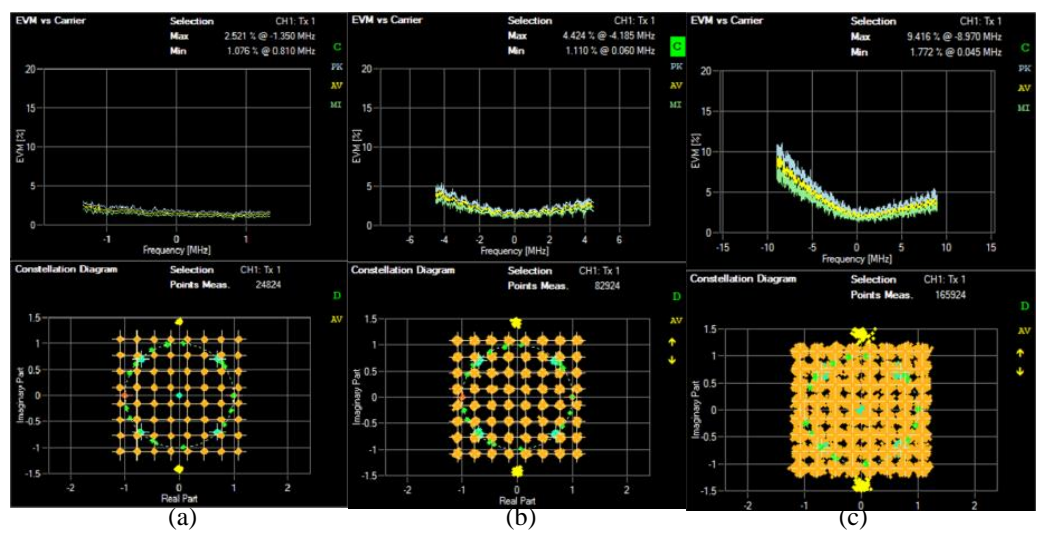

Fig. 8. Experimental test result for $2 \times 2$ LTE MIMO-enabled radio over $300 \mathrm{~m} \mathrm{MMF}$, using double sideband frequency translation technique. Central carrier frequency $=700 \mathrm{MHz}$ Upper: EVM vs. carrier frequency (normalized); Lower: constellation diagrams. (a)channel bandwidth=3MHz; (b)channel bandwidth $=10 \mathrm{MHz}$; (c)channel bandwidth $=20 \mathrm{MHz}$

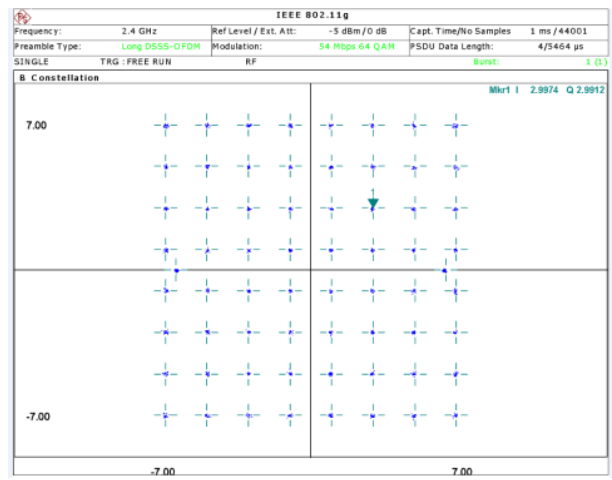

Fig. 9. Experimental test result for IEEE $802.11 \mathrm{~g}$ signal (54Mbps) transmitting along with $2 \times 2$ LTE MIIMO signals. Mean EVM=1.16\%.

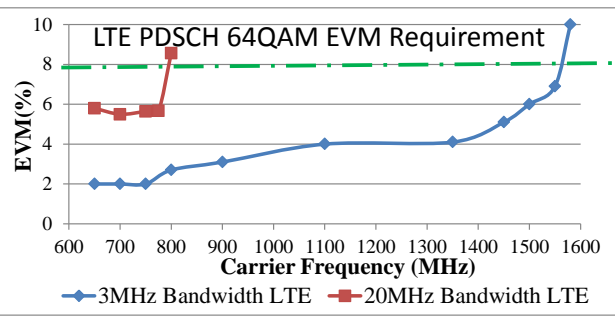

Fig.10. 2x2 LTE MIMO over 300m MMF. EVM performance versus carrier frequency

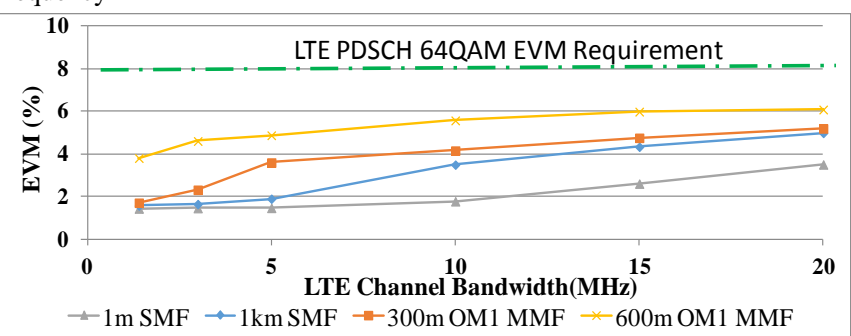

Fig.11. EVM comparison for four different types of optical fibers (carrier frequency $=700 \mathrm{MHz}$ )

in this range. The $\mathrm{S} 21$ amplitude-frequency and phase-frequency responses are logged to measure each element of the channel matrix of the $2 \times 2$ MIMO channels individually. Then the condition number of the MIMO channels can be calculated from maximum and minimum singular values of the channel matrix.

\section{EXPERIMENTAL RESULTS}

\section{A. $2 \times 2$ LTE along with IEEE 802.11g over RoF Link Test}

The system is tested using LTE channels at a 700MHz carrier frequency, and $3 \mathrm{MHz}, 10 \mathrm{MHz}$ and $20 \mathrm{MHz}$ channel bandwidths. In the experiment, two LTE channels are independently transmitted over 300m OM1 MMF using a temperature controlled DFB laser ( $\mathrm{RF}$ bandwidth $=2 \mathrm{GHz}$ ). Due to limitations of the test and measurement equipment, the EVM of each channel can only be measured independently, meaning that the recorded results are pessimistic compared to a full MIMO EVM since MIMO processing compensation is not employed. Fig. 8 shows EVM vs. carrier frequency curves and constellation diagrams. The average EVM measurement for $3 \mathrm{MHz}, 10 \mathrm{MHz}$ and $20 \mathrm{MHz}$ channel bandwidths are respectively $1.7 \%, 2.3 \%$ and $5.1 \%$. These values are well within the $8 \%$ EVM requirement for 64QAM in LTE Physical Downlink Shared Channel (PDSCH) standard. It can be seen in Fig. 8 (a) that for the $3 \mathrm{MHz}$ channel bandwidth, the EVM vs. carrier frequency curve remains relatively flat, while at the $20 \mathrm{MHz}$ channel bandwidth, the EVM at two sides of the band increases. This is due to the crosstalk caused by the amplitude mismatch. As illustrated in Fig.3. and equations $(11,13)$, the power equalizer needs to be changed to make the upper band and the lower band has the same amplitude. However, the frequency response of optical link changes with frequency. In the experiment, the power equalizer can only equalize the amplitude at a certain carrier frequency $(700 \mathrm{MHz})$. At frequencies around it, the amplitudes of two bands are no longer matching with each other. It is intended in future that the crosstalk caused by this be compensated by MIMO EVM compensation, which will be illustrated in the next section.

Simultaneously with the LTE channels, a channel of a 64QAM IEEE 802.11g signal is transmitted with the MIMO signals. It can be seen in Fig.9. that when the system input power is at the optimum level, the received Wi-Fi signal has a very low EVM ( 1.2\%) and the MIMO signals can exhibit the same EVM both with and without the $802.11 \mathrm{~g}$ signal, showing that the 802.11g signal and the MIMO signals do not influence each other. However, when the signal input power is high, intermodulation distortion between the Wi-Fi channel and the MIMO channels can occur due to nonlinear effects in the ROF link. It is experimentally shown that this effect can be minimized by carefully controlling the input power of the Wi-Fi signal. In the experimental layout, an attenuation of $10 \mathrm{~dB}$ is required to compensate the conversion loss of frequency mixer. In a practical system, an automatic gain control (AGC) unit could fulfill this function.

Fig.10 shows the results for the EVM test for $2 \times 2$ LTE MIMO over 300m OM1 MMF using different carrier frequency. The system has been tested using $+1 \mathrm{dBm}$ input signal power for optimum EVM results. Without MIMO signal processing, by tuning the power equalizer to suitable value, the system can operate up to a $1550 \mathrm{MHz}$ carrier frequency for $3 \mathrm{MHz}$ bandwidth LTE channels and a $800 \mathrm{MHz}$ carrier frequency for $20 \mathrm{MHz}$ bandwidth LTE channels. 
The RoF signals over MMF suffer from dispersion and distortion effects. Thus the link performance of a MMF RoF link should be worse than SMF link. An EVM performance comparison for four different types of optical fibers is shown in Fig.11. It can be seen that the proposed system is also suitable for both $1 \mathrm{~km}$ SMF and 600m OM1 MMF. EVM results for both are within LTE PDSCH standard requirement.

\section{B. Condition Number Test}

The unitary of the channel matrix can be measured by the condition number, which is expressed as

$$
\text { Condition number }=\frac{\sigma_{\max }(H)}{\sigma_{\min }(H)}=\left\|H^{-1}\right\|\|H\|
$$

in which $\sigma_{\max }(H)$ and $\sigma_{\min }(H)$ represents the maximum and minimum singular values of the channel matrix $\mathrm{H}$.

Fig.12. shows the measured channel matrix elements and the calculated condition numbers shown in $\mathrm{dB}$. The system is adjusted to operate at a carrier frequency of $700 \mathrm{MHz}$. It can be seen that at frequencies from $610 \mathrm{MHz}$ to $750 \mathrm{MHz}$, the condition number is lower than $10 \mathrm{~dB}$, which shows the system is well-conditioned and the EVM will be compensated in a $140 \mathrm{MHz}$ bandwidth if MIMO signal processing is used [18]. This bandwidth is enough to support intra-band carrier aggregation. For the case of inter-band carrier aggregation, multiple power equalizers need to be used.

Limited by the phase error of the 90-dgree hybrid couplers, the minimum value of the $\mathrm{h} 12$ and $\mathrm{h} 21$ parameters are not at the same frequency (Fig. 12). The minimum points for both the h12 and h21 curves occur when one of the equation (11) and (13) is satisfied and the intersection of the two curves occurs when $\theta$ is chosen to be an optimum value between $\sigma_{1}+\omega_{\mathrm{LO}} \Delta \mathrm{t}+n \pi$ and $\theta=\omega_{\mathrm{LO}} \Delta \mathrm{t}-\sigma_{2}+n \pi$, such that $\mathrm{h} 12$ and $\mathrm{h} 21$ are both small. At this point, the condition number is the lowest $(0.27 \mathrm{~dB})$ and the EVM can be well compensated by MIMO processing.

At the frequencies out of the bandwidth of power equalizer, the condition number increases and the system becomes ill-conditioned. In this case, the power equalizer needs to be adjusted.

\section{Noise Figure, SFDR and EVM Dynamic Range Test}

In the DSB system, when the upper and lower band signals are translated back to their original frequency, they sum in field, thus there will theoretically be a $6 \mathrm{~dB}$ signal power increase over a SSB system. In contrast the independent noise power will only be increased by $3 \mathrm{~dB}$. Experimentally, it is shown that, compared with the SSB system, the DSB system can increase the output signal power by $5.8 \mathrm{~dB}$ and output noise power by only $3.0 \mathrm{~dB}$, and thus the system NF is lowered from $46.0 \mathrm{~dB}$ to $43.2 \mathrm{~dB}$. This approximately agrees with theory and the $0.2 \mathrm{~dB}$ impairment can be attributed to the phase mismatch of the $90^{\circ}$ hybrid coupler.

The SFDR of the MIMO-enabled DSB frequency translation system is measured. In Fig.13, it is shown that by adjusting the pre-amplifier gain, the system SFDR increases from $101 \mathrm{~dB} \cdot \mathrm{Hz}^{2 / 3}$ to above $107 \mathrm{~dB} \cdot \mathrm{Hz}^{2 / 3}$, which is similar to the SFDR of the optical link alone $\left(110 \mathrm{~dB} \cdot \mathrm{Hz}^{2 / 3}\right)$. The degradation comes from the noise figure (NF) of the pre-amplifier.

In Fig.14, a comparison between the system using DSB

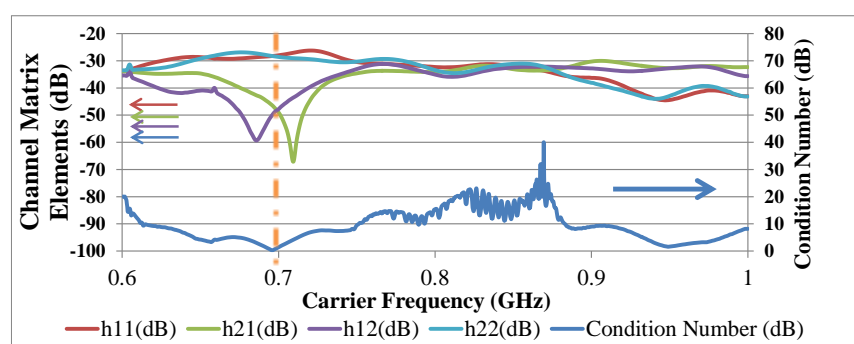

Fig.12. Measurement of channel matrix elements (phase is not shown) and calculated condition number vs. carrier frequency.

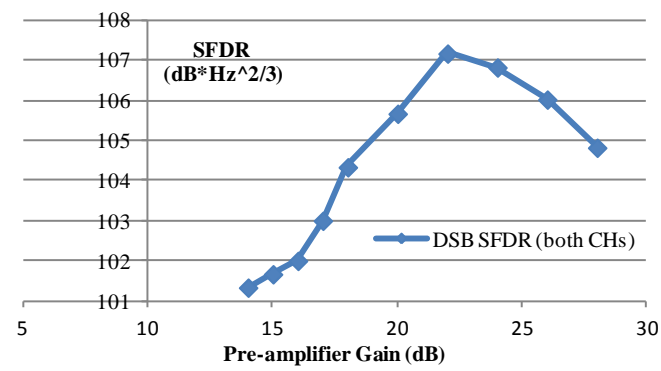

Fig.13. SFDR test for MIMO-enabled ROF system using DSB frequency

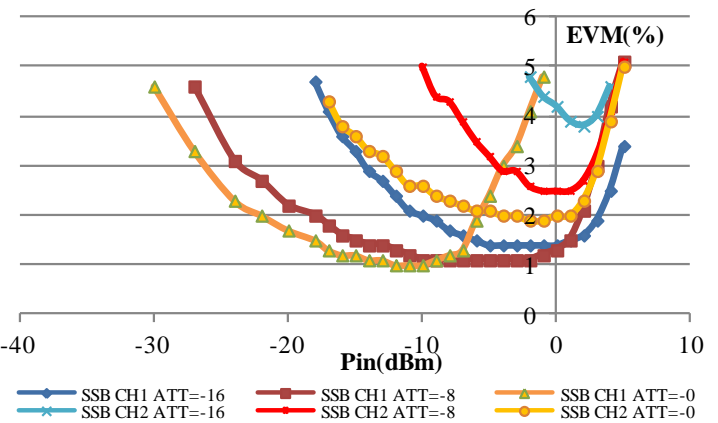

(a)

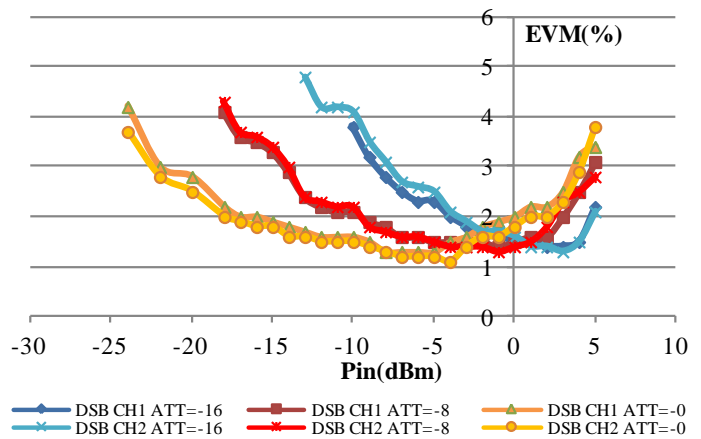

(b)

Fig.14. EVM dynamic range improvement of DSB frequency translation from SSB frequency translation, with different pre-amplifier gain (tested with two channels of 3.84 MHz QPSK signals, changing the attenuator following the pre-amplifier).

frequency translation and conventional SSB frequency translation is made when using $3.84 \mathrm{MHz}$ channel bandwidth. A variable attenuator is added at the output of the pre-amplifier to adjust the system dynamic range. It can be seen that in the SSB system, two MIMO channels always have different EVM curves. This is due to the bandwidth of the optical link - the upper band of the translated signal suffers from a much higher RF loss than the lower band, which results in a lower SNR. However, in the DSB system, as the two MIMO channels are orthogonally multiplexed, they occupy the same spectrum and 
have the same EVM performance. This leads to a much larger effective EVM dynamic range.

\section{CONCLUSIONS}

In this paper, we have demonstrated that the DSB translation technique is a feasible low-cost approach to transmit $2 \times 2$ MIMO-type LTE signals over a single optical link in unused wireless frequency bands, thus allowing multi-service DAS to support MIMO services for the first time. The simple frequency agnostic approach allows the key benefits of RoF to be maintained while supporting MIMO services.

Compared with the alternative SSB frequency translation technique, the proposed system has $\sim 3 \mathrm{~dB}$ higher SNR and has shown good linearity (when using 300m OM1 MMF, 3rd order SFDR $\sim 107 \mathrm{~dB} \cdot \mathrm{Hz}^{2 / 3}$ ). A condition number $<10 \mathrm{~dB}$ can be achieved over $140 \mathrm{MHz}$ bandwidth, which will allow intraband carrier aggregation with LTE services. Also, as both MIMO channels are orthogonally multiplexed with each other, they have similar EVM performance. As a result, compared with the SSB frequency translation, larger effective dynamic range can be achieved. In the wide-band signal transmission experiment, it is verified that the system can achieve a good EVM. The average EVM $(5.1 \%$ for $20 \mathrm{MHz}$ LTE and $1.2 \%$ for IEEE802.11g) is well within the requirements set by the standards. Together, the results demonstrate the feasibility of the DSB frequency translation technique in transmitting commercial LTE MIMO signals over indoor DASs using pre-existing installed fiber with improved performance compared to the SSB system.

\section{ACKNOWLEDGEMENT}

The authors would like to thank Tongyun Li, George S. D. Gordon and Adrian Wonfor for their support.

\section{REFERENCES}

[1] mobiForge "Global mobile statistics 2014 Part A: Mobile subscribers; handset market share; mobile operators, " may 2014. Internet: http://mobiforge.com/research-analysis/

global-mobile-statistics-2014-part-a-mobile-subscribers-handset-marketshare-mobile-operators?mT, May 16, 2014 [April 5, 2015].

[2] CISCO. "Cisco Visual Networking Index: Global Mobile Data Traffic Forecast Update, 2013-2018". Internet: http://www.cisco.com/c/en/us/solutions/collateral/service-provider/visua 1-networking-index-vni/white_paper_c11-520862.html, Feb. 5, 2015[April. 5, 2015]

[3] T. Norman, "Research forecast report-Wireless network traffic 2010-2015: forecasts and analysis," AnalysysMason, London, U.K., Tech. Rep.2010.

[4] M. Fabbri and , P. Faccin, "Radio over Fiber Technologies and Systems: New Opportunities," Transparent Optical Networks, 2007. ICTON '07. 9th International Conference on , vol.3, no., pp.230-233, 1-5 July 2007

[5] M.Katz. and F.Fitzek, WiMAX Evolution: Emerging Technologies and Applications. Chichester, U.K.: John Wiley \& Sons Ltd., pp389-390, 2009.

[6] Chin-Pang Liu; A. Seeds, "Transmission of MIMO radio signals over fibre using a novel phase quadrature double sideband frequency translation technique," Microwave photonics, 2008. jointly held with the 2008 asia-pacific microwave photonics conference. mwp/apmp 2008. international topical meeting on, pp.23-26, Sept. 9 2008-Oct. 32008

[7] Chin-Pang Liu; Seeds, A.J. "Transmission of Wireless MIMO-Type Signals Over a Single Optical Fiber Without WDM", Microwave Theory and Techniques, IEEE Transactions on, On page(s): 3094 - 3102 Volume: 58, Issue: 11, Nov. 2010
[8] J. Capmany, B. Ortega, A. Martinez, D. Pastor, M. Popov and P. Fonjallaz, "Multiwavelength single sideband modulation for WDM radio-over-fiber systems using a fiber grating array tandem device," Photonics Technology Letters, IEEE, vol.17, no.2, pp.471-473, Feb. 2005

[9] I. Seto, H. Shoki, and S. Ohshima, "Optical subcarrier multiplexing transmission for base station with adaptive array antenna," Microwave Theory and Techniques, IEEE Transactions on, vol.49, no.10, pp.2036,2041, Oct 2001

[10] G. Gordon, M. Crisp,; R. Penty, T. Wilkinson, and I. White, "Feasibility Demonstration of a Mode-Division Multiplexed MIMO-Enabled Radio-Over-Fiber Distributed Antenna System," Lightwave Technology, Journal of , vol.32, no.20, pp.3521-3528, Oct.15, 152014

[11] T. Li , M. Crisp, R. V. Penty and I. H. White "Low bit rate digital radio over fibre system", Int. Microw. Photon. Top. Meeting, 2009

[12] A. Chowdhury, H. Chien; S. Fan; J. Yu; G. Chang, "Multi-Band Transport Technologies for In-Building Host-Neutral Wireless Over Fiber Access Systems," Lightwave Technology, Journal of , vol.28, no.16, pp.2406-2415, Aug.15, 2010

[13] J. Zou; H. Chen; F. Huijskens, Z. Cao; E. Tangdiongga, and T. Koonen, "Demonstration of fully functional MIMO wireless LAN transmission over GI-MMF for in-building networks," Optical Fiber Communication Conference and Exposition and the National Fiber Optic Engineers Conference (OFC/NFOEC), 2013,pp.1-3, 17-21 March 2013

[14] M. Othman, L. Deng, X. Pang, J. Caminos, W. Kozuch, K. Prince, J. Jensen, I. Monroy, "Directly-modulated VCSELs for $2 \times 2$ MIMO-OFDM radio over fiber in WDM-PON," Optical Communication (ECOC), 2011 37th European Conference and Exhibition on , pp.1-3, 18-22 Sept. 2011

[15] L. Maksymiuk; J. Siuzdak, "Successful IEEE 802.11n 2-channel MIMO transmission over standard graded index multimode fiber in passband," Communications (QBSC), 2012 26th Biennial Symposium on, pp.15-18, 28-29 May 2012

[16] M. Morant, J. Prat, and R. Llorente, "Radio-Over-Fiber Optical Polarization-Multiplexed Networks for 3GPP Wireless Carrier-Aggregated MIMO Provision," Lightwave Technology, Journal of, vol.32, no.20, pp.3721-3727, Oct.15, 152014

[17] M. Ogita, S. Kimura, "Radio receiver having phase locked loop and automatic frequency control loop for stably maintaining local oscillator frequency of voltage-controlled local oscillator," U.S. Patent 4365349 A, December 21st, 1982.

[18] D. Tse and P. Viswanath, Fundamentals of Wireless Communication. Cambridge, U.K.: Cambridge Univ. Press, 2005. 